\title{
Influence of Prior Distributions and Fragility assessment methods in the estimation of the Magnitude of a Historical Seismic Event
}

\author{
Eduardo Charters MORAIS ${ }^{1}$, László Gergely VIGH ${ }^{1}$ and János KRÄHLING ${ }^{2}$ \\ ${ }^{1} \mathrm{BME}$, Department of Structural Engineering, Budapest, Hungary \\ ${ }^{2} \mathrm{BME}$, Department of History of Architecture and Monument Preservation, Budapest, Hungary
}

\begin{abstract}
The production of fragility functions describing the probable behaviour and damage on historical buildings is a key step in a method for the estimation of the magnitude of historical seismic events that uses a Bayes'. The fragilities are estimated by integrating the structural capacity with the seismic demand using either static methods, as the Capacity Spectrum Method (CSM), or dynamic methods, as Incremental Dynamic (IDA) and Multiple Stripes Analysis (MSA). Uncertainties in both resistance, demand, and distance and magnitude models propagate to the posterior magnitude distribution. The present paper studies the effect of uncertainties related both to the production of fragility functions and prior distributions, in the estimation of the magnitude of the 1763 Komárom earthquake (in historical Hungary). In the XVIII century most of the structures in the region were built of earth, adobe, clay or stone masonry, which is complex to model. While micro or detailed macro-modelling strategies are computationally costly, simplified macro-approaches are often more efficient, but require a pre-identification of the failure mode(s) and the determination of the backbone curve. For this study, a simplified macro-model of a Hungarian peasant house archetype is calibrated for CSM and IDA. The physical and geometrical uncertainties are incorporated in the fragilities using Monte-Carlo simulation. Prior magnitude and distance distributions are studied. The final magnitude estimates are presented and discussed.
\end{abstract}

\section{Introduction}

The most common methods for the estimation of the magnitude of historical seismic events $[1,2]$ belong to the domain of seismology, using interdisciplinary knowledge of both geophysicists and historians [3]. Another method applies the background of Probabilistic Seismic Hazard Analysis (PSHA) and uses historical seismic records of damage to translate the nonlinear structural damage into fragility curves [4], obtained either by dynamic methods as Incremental Dynamic and Multiples Stripes Analysis (IDA \& MSA) [5] or by static methods as the Capacity Spectrum Method (CSM) [6]. These methods integrate the structural capacity with the seismic demand - usually correlated - and their respective uncertainties, to produce the damage points for fragility function fitting. The magnitude estimation method [4] combines the historical data of the seismic damage with the fragility functions to produce the likelihood of a seismic event $(E)$. Then, it applies the total probability and Bayes' theorems using an attenuation model, or ground motion prediction equation (GMPE), and prior distance ( $r$ or $R_{e p}$ ) and magnitude ( $m$ or $M_{w}$ ) distributions - details in subsection 2.3. As a result, both capacity and demand related uncertainties, the GMPE(s) and prior $m$ and $r$ distributions contribute to the reliability of the final magnitude estimates.
The present paper focus both on the impact fragility functions, for historical buildings affected by the 1763 Komárom (nowadays Komárno, Slovakia) earthquake one of the most destructive in Hungarian history -, and on the weight of assumptions in the final magnitude estimates. Therefore, the modelling tools, their efficiency, as well as the main uncertainty sources in the magnitude estimation method are described and studied. A general overview of the methodologies in study is provided in section 2 . In section 3 the empirical capacity curves are studied for a set of historical adobe unreinforced masonry wall panels from a one-storey peasant house archetype [7, 8]. A simplified macro-model that uses a multilinear spring [9] is calibrated for IDA, incorporating physical uncertainties associated with the mechanical parameters defined in literature [10-13], using Monte-Carlo simulation (MCS). CSM is also here implemented with the help of MCS on graphic based approach. In section 4 the weight of the fragilities, GMPE and prior distributions parameters is balanced. The methodologies and results are discussed, and conclusions presented.

\section{Methodologies}

The methodology presented here is interdisciplinary, requiring the interpretation of historical sources of 
damage and structural data related to the 1763 Komárom earthquake, as well as features of the dynamic modelling and analysis of the historical structures.

\subsection{The 1763 Komárom earthquake}

The 1763 Komárom earthquake, in the $28^{\text {th }}$ of June, was one of the most destructive in historical Hungary. Although, the estimation of its magnitude is questioned by distinct estimates, in between 5.70 and 6.50 [1, 2, 13]. Its destructive effect led to the production of a vast quantity of documents and depictions, as that in figure 1. In the aftermath, out of 1169 houses in total, 279 completely collapsed, 353 partially collapsed, 213 needed expensive repair and 219 cheap repair [2]. Historical sources identify the most damaged buildings as two storey rigid buildings, made of burnt clay bricks, rather than simple and more flexible peasant houses, made of earthen or adobe masonry. The resulting depictions also helped to identify common failure modes as shear failure [11] enabling the simplification of the modelling work.

Furthermore, the interpretation of the damage sources resulted on the need for the systematized generation of historical building archetypes representing the regional features of XVIII c. West of Hungary [7]. This happened mainly due to the lack damage descriptions of existing representative buildings. Therefore, Archetypes were generated for different building categories, the most representative being tax-payer's houses, as the peasant house [8] represented in figure 2 :
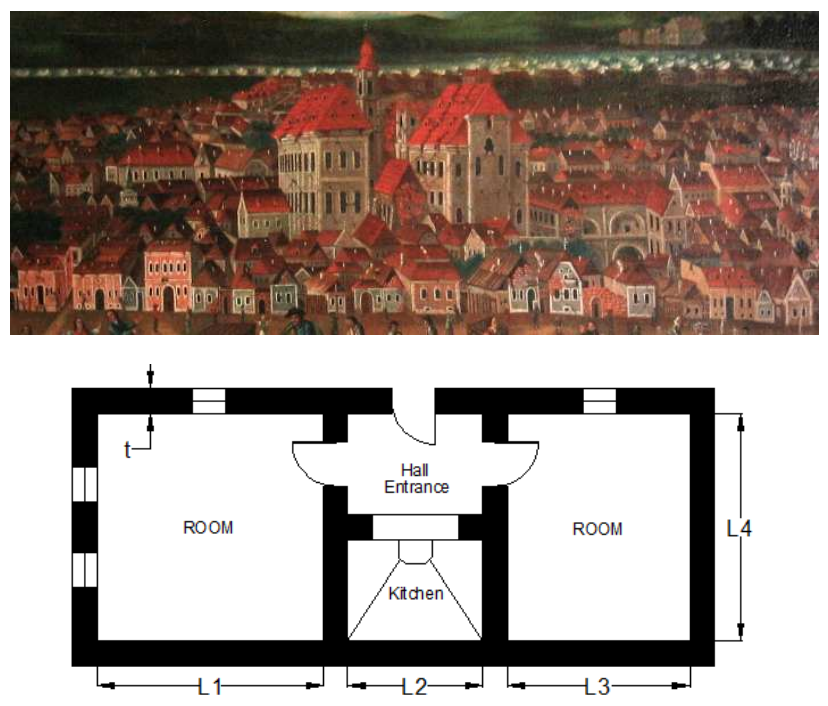

Fig. 1. Anonymous depiction of the damage in the city of Komárom after the 1763 earthquake (up) \& plan of a XVIII century Hungarian peasant house archetype, from [5].

\subsection{Fragility functions}

The fragility functions are produced using dynamic or static structural analysis methods such as IDA and CSM and describe the conditional probability of occurrence of a certain damage measure $D M$ implying one or more damage states $d s_{i}\left(D M \rightarrow d s_{i}\right)$ given a set of parameters $\theta$ - an intensity measure im (may be PGA, PGV, $\mathrm{S}_{\mathrm{a}}(\mathrm{T})$ ), $m$ and $r$. They are usually assumed as lognormally fitted
$\mathrm{CDF}$ functions and described in terms of intensity im, with medians $\mu_{d s i}$ and standard deviations $\beta_{d s i}$ :

$P\left(d s_{i} \mid \theta\right)=P\left(d s_{i} \mid i m, m, r\right)=\Phi\left(\ln \left(x / \mu_{d s i}\right) / \beta_{d s i}\right)$

\subsection{The Magnitude estimation method}

The magnitude estimation method is described in detail in [4] and its flowchart is presented in figure 2:

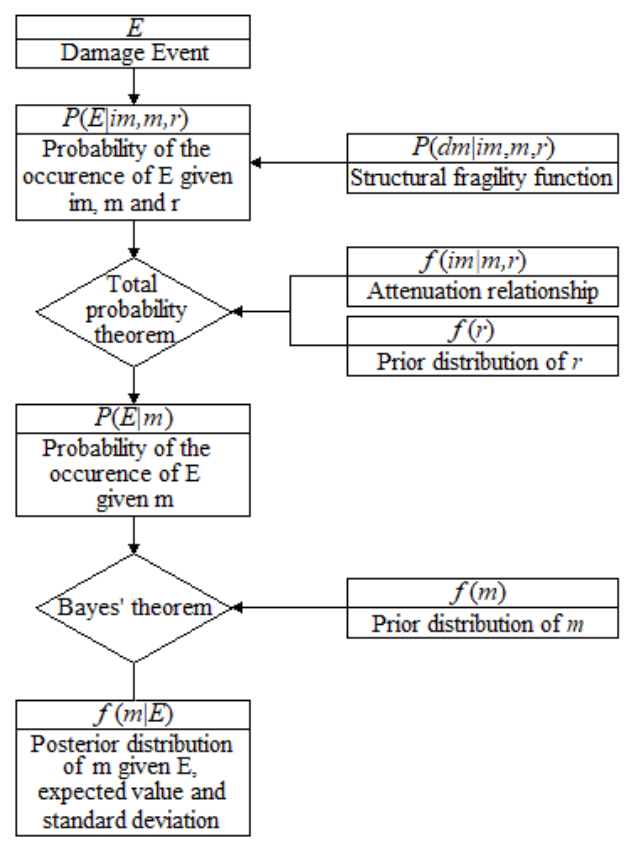

Fig. 2. Flowchart of the magnitude estimation method, from [4].

The original form of the problem consists of stating an occurred seismic event $E$, characterized by an intensity measure $i m$, or and $m$ and $r$, which caused damage in structures, represented by a damage measure $D M$. So, ' $E$ caused $D M$ ' implies here a certain probability of occurrence $P(E \mid i m, m, r)$ described by the combination of fragility functions in the form $P(D M \mid i m, m, r)$ with the number of structures $n_{d m}$ under the damage measures $D M$. Afterwards, the total probability theorem enables to integrate the conditional probability $P(E \mid i m, m, r)$ with the attenuation relationship $f(i m \mid m, r)$ and prior distribution $f(r)$, in order to calculate the conditional probability of $E$ given $m, P(E \mid m)$. Then, the posterior distribution $f(m \mid E)$ is achieved using the Bayes' theorem together with a prior distribution $f(m)$. These operations may be synthesized by expressions (1) and (2), respectively:

$$
\begin{aligned}
& P(E \mid m)=\iint P(E \mid \text { im, } m, r) \times f(\text { im } \mid m, r) \times f(r) d i m d r \\
& f(m \mid E)=P(E \mid m) \times f(m) / \int P(E \mid m) \times f(m) d m
\end{aligned}
$$

Despite the use of dynamic methods, applying CSM directly with the help of a GMPE leads to the $i m$ as a function of $m$ and $r$, in the form $\operatorname{im}(m, r)$, resulting that the conditional probability of $E$ may assume the form $P(E \mid m, r)$. Thus expression (2) could be simplified:

$$
P(E \mid m)=\int P(E \mid m, r) \times f(r) d r
$$

The direct use of the $m$ and $r$ inputs via GMPE describes a probability surface of the type $P(E \mid m, r)$. It is 
also an economic alternative to directly select the $m$ values associated with damage states, if the $r$ values are sampled from the prior $f(r)$, simplifying the numerical integration, resulting on the sole use of expression (3).

\section{Static and dynamic based analysis and fragilities}

The adopted modelling and uncertainty incorporation strategies and analysis approaches CSM and IDA are here described. Deeper considerations and details about the methods can be found elsewhere $[5,6,11,14]$.

\subsection{Complex vs simplified modelling}

The modelling of historical buildings is a complex issue. The increase of computation capacity has allowed the production of increasingly sophisticated modelling tools, since the 1990's. Different finite and discrete element (FEM and DEM) based approaches, enabled multi-level modelling strategies: detailed micro-, simplified micro- and detailed macro-modelling [15]. Although the computation cost decreases from micro to macro strategies, it is still costly to produce detailed macro-models capable of incorporating at the same time, and using dynamic time-history analysis - IDA or MSA, uncertainties in seismic demand and structural capacity. An alternative is to use a simplified macro-modelling strategy with an SDOF system adapted to simulate the inplane behaviour of a wall panel by calibration of a nonlinear spring [9] (figure 3, left). In turn, the $F-\delta$ diagram may be achieved by predicting the $\mathrm{F}-\delta$ backbone [16] or by using more detailed modelling-levels [15]. This strategy enables to study uncertainties in both structural capacity - as function of compressive strength $f_{m}$, Young modulus $E_{m}$, internal friction $\mu$ and other geometrical parameters $\theta_{G}, R\left(f_{m}, E_{m}, \mu, \theta_{G}\right)$ - and the seismic demand - as function of $m, r$ and other parameters related to fault and soil types $\theta_{S F}, D\left(m, r, \theta_{S F}\right)$.
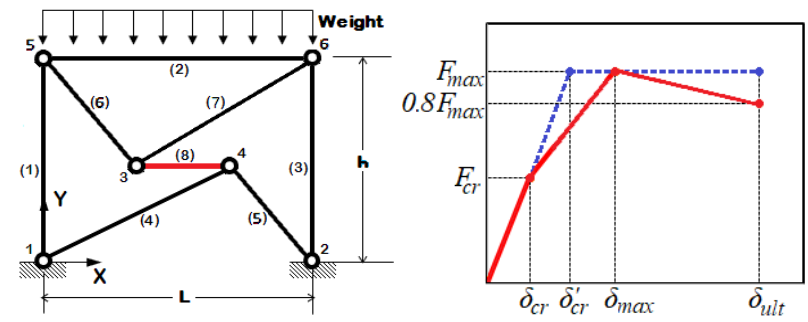

Fig. 3. SDOF isostatic model with a nonlinear string (left) and respective bi- and tri-linear models (right), from $[11,16]$.

\subsection{CSM based fragilities}

The CSM involves both the determination of the pushover based capacity curve and demand spectrum, translated in terms of spectral displacements $\left(S_{d}\right)$ and spectral accelerations $\left(S_{a}\right)$. The intersection gives the performance point (figure 4). Details are elsewhere [6, 11]. For the analysis, the median values $E_{m}=0.209 \mathrm{MPa}$, $\mu=0.45$ and $T_{s s}=1.70 \mathrm{MPa}$ [16] are adopted with lognormal distribution, with $25 \%, 19 \%$ and $25 \%$ of covariance, respectively. Damage is modelled based on [17]. The final fragilities, presented in table 1 . In previous developments, the description of the fragilities has been affected by the $m$ and $r$ value ranges of interest, resulting that $d s_{1}$ resulted to be well described in detriment of $d s_{2}$, $d s_{3}$ and $d s_{4}$ [17]. The previous code has been improved in order to achieve more complete descriptions.

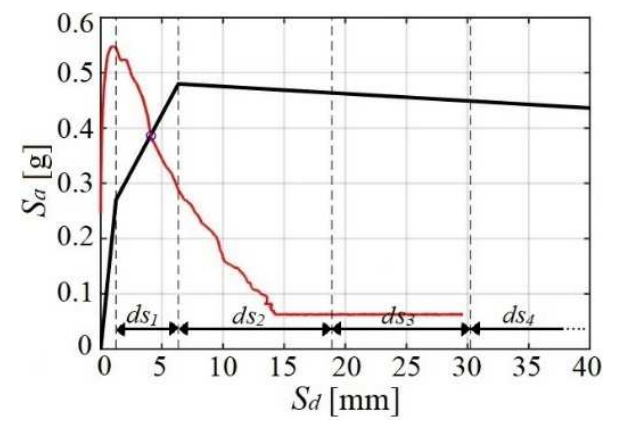

Fig. 4. CSM intersection of structural capacity with the seismic demand in the $S_{a}-S_{d}$ axis, based on [11].

Table 1. Fragility moments mean and standard deviation PGA's, in [g], for the walls 1-to-6, from CSM.

\begin{tabular}{|c|c|c|c|c|c|c|}
\hline Wall & $\mathbf{1}$ & $\mathbf{2}$ & $\mathbf{3}$ & $\mathbf{4}$ & $\mathbf{5}$ & $\mathbf{6}$ \\
\hline $\boldsymbol{\mu}_{\boldsymbol{d s} \mathbf{1}}$ & 0.21 & 0.21 & 0.44 & 0.23 & 0.24 & 0.33 \\
\hline $\boldsymbol{\beta}_{\boldsymbol{d s} \mathbf{1}}$ & 0.22 & 0.37 & 0.16 & 0.27 & 0.28 & 0.22 \\
\hline $\boldsymbol{\mu}_{\boldsymbol{d s} \mathbf{2}}$ & 0.37 & 0.38 & 0.83 & 0.42 & 0.43 & 0.57 \\
\hline $\boldsymbol{\beta}_{\boldsymbol{d s} \mathbf{2}}$ & 0.18 & 0.09 & 0.21 & 0.14 & 0.14 & 0.16 \\
\hline $\boldsymbol{\mu}_{\boldsymbol{d s} \mathbf{3}}$ & 0.51 & 0.48 & 1.26 & 0.55 & 0.57 & 0.81 \\
\hline $\boldsymbol{\beta}_{\boldsymbol{d s} \mathbf{3}}$ & 0.08 & 0.12 & 0.04 & 0.08 & 0.08 & 0.08 \\
\hline $\boldsymbol{\mu}_{\boldsymbol{d s} \mathbf{4}}$ & 0.69 & 0.72 & 1.61 & 0.84 & 0.87 & 1.19 \\
\hline $\boldsymbol{\beta}_{\boldsymbol{d s} \mathbf{4}}$ & 0.15 & 0.15 & 0.13 & 0.19 & 0.19 & 0.17 \\
\hline
\end{tabular}

\subsection{IDA based fragilities}

The main idea of IDA is the scaling of the selected $\mathrm{im}$ values, in the present case peak ground acceleration (PGA), until the collapse of the structure (figure 5). The analysis was carried out with the same tri-linear model and formulation, but using 30 seismic records selected for the region of Komárom [5].

The final IDA based fragilities, presented in table 2, incorporate not only the physical uncertainties, as CSM application in sub-section 3.2, but also the uncertainty related to the frequency and duration associated to the seismic records. Which is not featured by CSM.

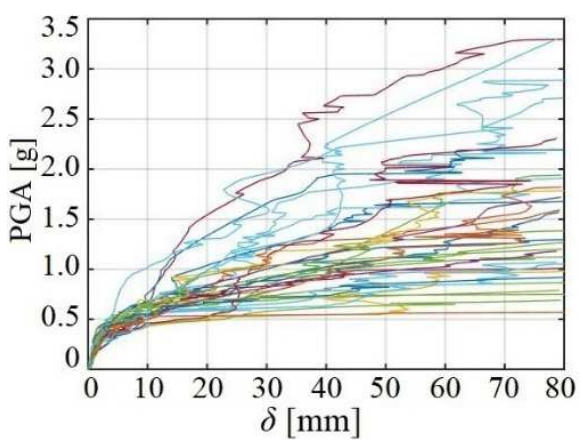

Fig. 5. IDA results for wall 4, based on [11]. 
Table 2. Fragility moments mean and standard deviation PGA's, in [g], for the wall panels 1-to-6, from IDA.

\begin{tabular}{|l|c|c|c|c|c|c|}
\hline Wall & $\mathbf{1}$ & $\mathbf{2}$ & $\mathbf{3}$ & $\mathbf{4}$ & $\mathbf{5}$ & $\mathbf{6}$ \\
\hline $\boldsymbol{\mu}_{\boldsymbol{d m} \mathbf{1}}$ & 0.23 & 0.19 & 0.18 & 0.21 & 0.20 & 0.21 \\
\hline $\boldsymbol{\beta}_{\boldsymbol{d m} \mathbf{1}}$ & 0.34 & 0.26 & 0.24 & 0.23 & 0.22 & 0.22 \\
\hline $\boldsymbol{\mu}_{\boldsymbol{d m} \mathbf{2}}$ & 0.37 & 0.57 & 0.30 & 0.43 & 0.42 & 0.41 \\
\hline $\boldsymbol{\beta}_{\boldsymbol{d m} \mathbf{2}}$ & 0.26 & 0.30 & 0.22 & 0.19 & 0.19 & 0.18 \\
\hline $\boldsymbol{\mu}_{\boldsymbol{d m} \mathbf{3}}$ & 0.68 & 0.69 & 1.00 & 0.70 & 0.70 & 0.83 \\
\hline $\boldsymbol{\beta}_{\boldsymbol{d m} \mathbf{3}}$ & 0.20 & 0.32 & 0.39 & 0.29 & 0.30 & 0.36 \\
\hline $\boldsymbol{\mu}_{\boldsymbol{d m} \mathbf{4}}$ & 0.76 & 0.91 & 1.29 & 0.87 & 0.88 & 1.02 \\
\hline $\boldsymbol{\beta}_{\boldsymbol{d m} \mathbf{4}}$ & 0.25 & 0.35 & 0.50 & 0.36 & 0.36 & 0.41 \\
\hline
\end{tabular}

\subsection{FEMA HAZUS based fragilities}

FEMA Hazus technical manual [10] makes use of CSM and prescribe $F-\delta$ diagrams for low and mid-rise buildings (URML \& URM, respectively). Therefore, the fragility medians are determined by the $R-D$ intersection. The standard deviation is calculated using expression (5).

$\beta_{d s}=\left(\operatorname{CONV}\left[\beta_{C}, \beta_{D}\right]^{2}+\beta_{T, d s}{ }^{2}\right)^{1 / 2}$

Where $\beta_{d s}$ is the lognormal standard deviation that describes the total variability for structural damage state, $d s_{i}, \beta_{C}=0.30$ and is associated with the capacity, $\beta_{D}=0.50$ and is associated with the demand, and $\beta_{T, d s}=0.40$ is associated with the damage state threshold. Assuming the previous values from [10] the total variability $\beta_{d s}=0.43$ was achieved. The median values are described with the help of a scaled demand $D\left(m, r, \theta_{S F}\right)$ given by the attenuation from [18], averaged for the intervals of interest $m \in[5,8]$ and $r \in[0,20]$, with strike-slip fault type and soft soil. The results are presented in table 3 .

The elements that transit from CSM and IDA to the magnitude estimation are the fragilities. Despite the small differences evidenced here between CSM and IDA, it is a question how much uncertainty may be reduced with the use of more advanced simulation methods. Other studies suggest that model uncertainties may raise to 0.50 on force side and 0.90 on deformation side [11]. FEMA P695 [12] provides ratings and uncertainty values for different sources: design-requirements, test data and modelling related uncertainties. These are sorted from $\beta_{\text {Mod }}=0.10$ to 0.50 , from high to low 'completeness and robustness', and from high to poor 'confidence in basis of design requirements', but these many sources may not guarantee improvements of the final magnitude estimates.

Table 3. Fragility medians in terms of PGA [g] using FEMA HAZUS suggested capacities for damage states $d s_{i}$.

\begin{tabular}{|c|c|c|c|c|}
\hline- & $\boldsymbol{d s}_{\boldsymbol{1}}$ & $\boldsymbol{d s}_{\boldsymbol{2}}$ & $\boldsymbol{d s}_{\boldsymbol{3}}$ & $\boldsymbol{d s}_{\boldsymbol{4}}$ \\
\hline Low-rise & 0.21 & 0.35 & 0.80 & 1.12 \\
\hline Mid-rise & 0.19 & 0.26 & 0.56 & 1.10 \\
\hline
\end{tabular}

\section{Magnitude estimates}

The reliability of the posterior magnitude distribution and estimates depends not only on the fragilities but also on the quality and completeness of the historical data and of the prior distributions and attenuation relationship(s).
In this section the impact of the fragilities, the priors and the historical data on structures is evaluated.

\subsection{Sensitivity study}

The calibration example, developed in [4], consisted on the application of the method to the 1994 Northridge (WES-US) instrumental earthquake using damage data on buildings associated to the FEMA HAZUS fragilities [10]. The code was utilized to study the input parameters and the sensitivity of the method, with input variables and uncertainty sources in: (1) the fragility moments' $\mu_{d s i}$ and $\beta_{d s i}$ (2) the GMPE's $\mu_{I M}$ and $\beta_{I M}$, (3) the historical records based $d s_{i}$ data, (4) the prior distance data, $f(r)$ type and bounds, and (5) the prior magnitude data, $f(m)$ type and bounds. For immediate purpose of studying the first two topics, the prior $f(m)$ is maintained as uniform, the prior $f(r)$ is tested in the interval 0 to $20 \mathrm{~km}$, the $d s_{i}$ array [105 444235 27], as in [19], and the fragilities in table 3, corresponding to a low-rise building are used. The results show a plateau in the magnitude estimate $\mu_{M}$ and respective standard deviation $\beta_{M}$, associated to $\beta_{d s i}$ and $\beta_{I M}$ values bigger than 0.3 and 0.5 , respectively (figure 6). Both $\mu_{M}$ and $\beta_{M}$ are less sensitive to $\beta_{d s i}$ than to $\beta_{I M}$, as the sensitivity factors of 0.11 and 0.99 were approximately estimated in regards to $\mu_{M}$, and 0.33 and 0.99 in regards to $\beta_{M}$. This suggests that the ground motion uncertainties, rather than the fragility related ones, have a considerable effect on the uncertainty in the magnitude estimates.

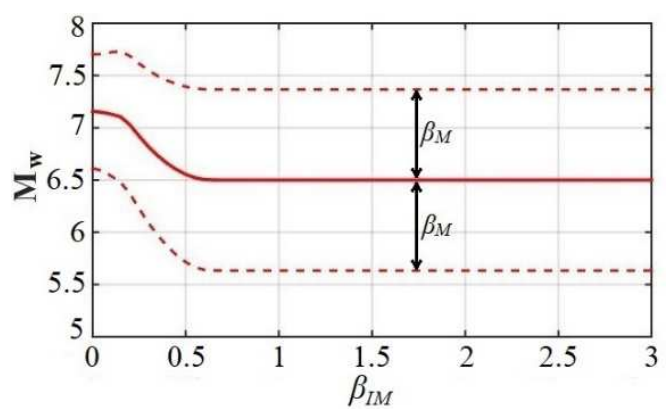

Fig. 6. Variation of $\mu_{M} \pm \beta_{M}$ with varying $\beta_{I M}$.

\subsection{Prior magnitude and distance distributions}

In order to achieve the prior distribution of magnitude $f(m)$, historical earthquakes occurred in the regions of Mór, Győr and Komárno (Komárom) between 1615 and 1886, were selected [13]. Their magnitudes were computed as normally distributed with means ranging between 3.8 and 5.7 and standard deviations between 0.33 and 0.50 , resulting in a lognormal prior $f(m)$.

Afterwards, the magnitude data was truncated for the range of interest $-M_{w}$ from 5 to 8 - creating a truncated lognormal prior $f(m)$. In figure 7 , six possible $m$ priors are shown. They are adapted for the region affected by the 1763 event. The latter were fitted for historical seismic events in the ranges $3.8 \leq \mathrm{M}_{\mathrm{w}} \leq 5.7$ for $f_{m}{ }^{I I}$ and $5.0 \leq \mathrm{M}_{\mathrm{w}} \leq 5.7$ for $f_{m}{ }^{I I I}$. These are lognormally distributed and defined by the moments $4.07 \pm 0.17$ and $5.41 \pm 0.06$, respectively. The prior $f_{m}{ }^{I}$ has a uniform distribution in $5.0 \leq \mathrm{M}_{\mathrm{w}} \leq 8.0$ and the $f_{m}{ }^{I V}$ to $f_{m}{ }^{V I}$ priors are described in section 4.3. The 
suggestions for prior distribution of distance $f(r)$ from [4] are studied together with the sensitivity of the magnitude estimates to the selection of two sets of bounds prior data suggests, in the ranges 0 to $20 \mathrm{~km}$, and 10 and $12 \mathrm{~km} \mathrm{[2],}$ from Komárom. The priors $f_{r}^{I}$ to $f_{r}^{I I I}$ are represented in figure 8 in the range 0 to $20 \mathrm{~km}$.

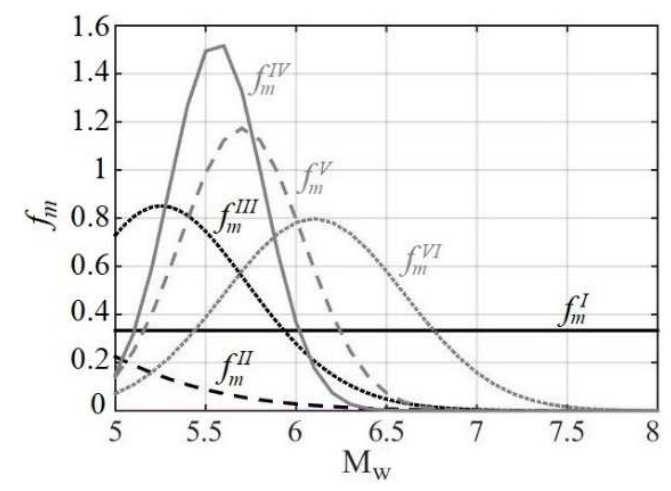

Fig. 7. Prior distributions of magnitude in the range of interest $\left(M_{w}\right.$ between 5 and 8$)$.

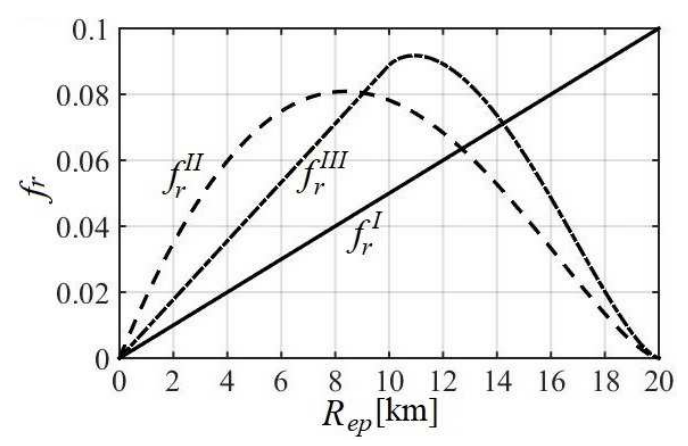

Fig. 8. Prior distributions of distance in the range of interest $\left(R_{e p}\right.$ between 0 and $20 \mathrm{~km}$ ), based on [4].

\subsection{Comparison of magnitude estimates}

The fragilities in 3.2 and 3.3 from CSM and IDA were averaged in order to re-establish new fragility moments, resulting in the values in table 4. Afterwards, in order to compare the magnitude estimates for the different sets of fragilities, damage data and prior distributions, a strategy was set, firstly to compare with previous estimations [19] (table 5, Archetype), secondly, to test the damage descriptions stating the resilience of smaller adobe houses rather than bigger clay buildings (tables 5, respectively), and finally to draw a comparison between the Archetype CSM and IDA and the FEMA HAZUS based fragilities (in table 6). In the last group the $R_{e p}$ range is restricted and three more normally distributed magnitude priors, representing previous magnitude estimations for the 1763 event, are studied: $f_{m}{ }^{I V}=5.56 \pm$ $0.26, f_{m}{ }^{V}=5.70 \pm 0.34$ and $f_{m}{ }^{V I}=6.10 \pm 0.50$ [13] (figure 7).

Table 4. Averaged fragility moments $\mu_{L N}$ and $\beta_{L N}$, in terms of PGA [g], for the peasant house walls 1-to-6.

\begin{tabular}{|c|c|c|c|c|}
\hline- & $\boldsymbol{d s}_{\boldsymbol{1}}$ & $\boldsymbol{d s}_{\boldsymbol{2}}$ & $\boldsymbol{d s}_{\mathbf{3}}$ & $\boldsymbol{d s}_{\boldsymbol{4}}$ \\
\hline $\boldsymbol{\mu}_{\boldsymbol{L N}}$ & 0.23 & 0.44 & 0.65 & 0.95 \\
\hline $\boldsymbol{\beta}_{\boldsymbol{L N}}$ & 0.35 & 0.31 & 0.41 & 0.38 \\
\hline
\end{tabular}

Table 5. Expected $\mu_{M} \pm \beta_{M}$ for distance $0 \leq R_{e p} \leq 20$, magnitude priors $f_{m}{ }^{I}$ to $f_{m}{ }^{I I I}$, and $d s_{i}=\left[\begin{array}{ll}10544 & 423527\end{array}\right]$ for the Archetype

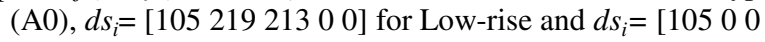

353 279] for Mid-rise unreinforced masonry (URML \& URM).

\begin{tabular}{|c|c|c|c|c|}
\hline - & - & $f_{r}^{I}$ & $f_{r}^{I I}$ & $f_{r}^{I I I}$ \\
\hline \multirow{3}{*}{ \& } & $f_{m}{ }^{I}$ & $6.81 \pm 0.73$ & $6.64 \pm 0.81$ & $6.69 \pm 0.78$ \\
\hline & $f_{m}{ }^{I I}$ & $5.72 \pm 0.51$ & $5.57 \pm 0.45$ & $5.63 \pm 0.46$ \\
\hline & $f_{m}^{I I I}$ & $5.60 \pm 0.30$ & $5.54 \pm 0.28$ & $5.57 \pm 0.28$ \\
\hline \multirow{3}{*}{$\underset{\mathfrak{S}}{\$}$} & $f_{m}{ }^{I}$ & $6.72 \pm 0.76$ & $6.59 \pm 0.83$ & $6.62 \pm 0.81$ \\
\hline & $f_{m}^{I I}$ & $5.66 \pm 0.48$ & $5.53 \pm 0.43$ & $5.57 \pm 0.44$ \\
\hline & $f_{m}{ }^{I I I}$ & $5.59 \pm 0.29$ & $5.52 \pm 0.28$ & $5.54 \pm 0.28$ \\
\hline \multirow{3}{*}{$\underset{S}{\$}$} & $f_{m}{ }^{I}$ & $7.15 \pm 0.55$ & $7.09 \pm 0.58$ & $7.15 \pm 0.55$ \\
\hline & $f_{m}^{I I}$ & $6.33 \pm 0.46$ & $6.27 \pm 0.44$ & $6.33 \pm 0.46$ \\
\hline & $f_{m}{ }^{I I I}$ & $5.94 \pm 0.21$ & $5.93 \pm 0.21$ & $5.94 \pm 0.28$ \\
\hline
\end{tabular}

Table 6. Expected $\mu_{M} \pm \beta_{M}$ for distance $10 \leq R_{e p} \leq 12$ and

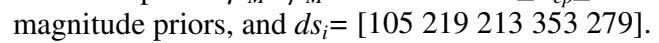

\begin{tabular}{|c|c|c|c|c|c|c|}
\hline- & $f_{m}{ }^{\boldsymbol{I}}$ & $\boldsymbol{f}_{m}{ }^{\boldsymbol{I I}}$ & $\boldsymbol{f}_{m}{ }^{\boldsymbol{I I I}}$ & $\boldsymbol{f}_{m}{ }^{\boldsymbol{V}}$ & $\boldsymbol{f}_{\boldsymbol{m}}{ }^{\boldsymbol{V}}$ & $\boldsymbol{f}_{\boldsymbol{m}}{ }^{\boldsymbol{I I}}$ \\
\hline $\boldsymbol{\mu}_{\boldsymbol{M}}$ & 7.11 & 6.55 & 6.30 & 6.24 & 6.34 & 6.57 \\
\hline $\boldsymbol{\beta}_{\boldsymbol{M}}$ & \pm 0.52 & \pm 0.34 & \pm 0.14 & \pm 0.11 & \pm 0.15 & \pm 0.29 \\
\hline
\end{tabular}

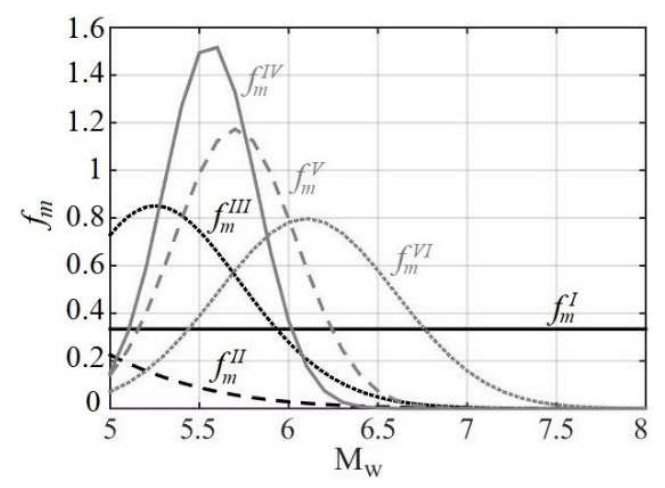

Fig. 9. Posterior magnitude distributions for table 8, considering the priors $f_{m}{ }^{I}$ to $f_{m}{ }^{V I}$.

\subsection{Discussion of the results}

The sensitivity study in subsection 4.1 suggest the relevance of $\beta_{I M}$ rather than $\beta_{d s i}$ for both $\mu_{M}$ and $\beta_{M}$ values, but no effect for $\beta_{I M}$ bigger than approximately 0.60 . According to subsection $4.3, \beta_{M}$ may be reduced by effect of the magnitude priors, as shown by the use of $f_{m}^{I I I}$ to $f_{m}{ }^{V}$ (tables 5 to 8 ). For the studied $R_{e p}$ ranges, the use of different $f_{r}$ distributions has a small effect on results, less than $2 \%$ on both $\mu_{M}$ and $\beta_{M}$. Otherwise, the application of the priors $f_{m}{ }^{I I}$ to $f_{m}{ }^{V I}$ highly affects the $\mu_{M}$ values, if compared to the uniform prior $f_{m}{ }^{I}$. A possible reading is that the damage based estimation points to high $\mu_{M}$ values, as can be seen in tables 5 and 6 first rows, while prior information on the seismicity of the region contributes to lower this value. This contribution can be seen as an influence of the median values and inversely proportional to the standard deviation (figure 9).

Additionally, the selected GMPE [18] with $m=8.0$ and $r=0 \mathrm{~km}$ produces a $P G A=0.42 \pm 0.28 \mathrm{~g}$. Consequently, when the likelihood $P(E \mid i m, m, r)$ of the event is estimated, the probability associated to damage states $d s_{3}$ and $d s_{4}$ for higher PGA's becomes also high, resulting in a poor description of the posterior distribution (figure $9, f_{m \mid E}^{I}$ ). 
Another open point here is the small difference in the fragilities utilized in the analysis, which resulted in small differences between the estimates associated to the two buildings in comparison [8, 10] (table 6). The effect of the variation of fragilities' median values was tested by incrementing them with additional $0.2 \mathrm{~g}$, which resulted in an increase of $10 \%$ in $\mu_{M}$.

\section{Conclusions}

The present paper focusses on how fragility and GMPE related uncertainties, and assumptions based on prior information, impact the final magnitude estimates, extending earlier works on the simplified assessment of the behaviour of historical unreinforced masonry walls and magnitudes for the 1763 Komárom earthquake.

Despite the poor description of the im with CSM, the experimental capacity curves and the FEMA HAZUS manual led to similar fragility moments, although the fragility medians rather than their standard deviations are relevant for the final magnitude estimates. Otherwise, the standard deviation associated to the GMPE has a high impact on the expected value of magnitude and respective standard deviation. The fact that the chosen GMPE leads to limited descriptions for higher im values - and thus for higher magnitudes - has been confirmed by the CSM fragilities and the posterior distribution $f_{m \mid E}^{I}$ in figure 9. The expected magnitude and standard deviation are affected mainly by the prior distribution of magnitude rather than by the prior distribution of distance, despite the small studied distance ranges (tables 5 and 6). In addition, the sole consideration of the damage analysis resulted in higher order magnitude estimates (6.6 7.2), while the use of magnitude priors resulted in lower order magnitude estimates of (5.5 6.6). The full use of prior knowledge (table 6 , figure 9) led to magnitude expected values in between 6.2 and 6.6.

Improvements may be achieved with further work with other numerical modelling tools and other building archetypes that may be used to produce valid fragilities. Other prior distributions of magnitude and distance may also be tested and discussed in more detail. As to the use of different GMPE(s) associated in a logic tree may improve the im descriptions.

\section{Acknowledgements}

Proper acknowledgements to CAPES for allowing the present research (Process No. 9178-13-9). This paper was also supported by the János Bolyai Research Scholarship of the Hungarian Academy of Sciences.

\section{References}

1. G. Szeidovitz, Earthquakes in the region of Komárom, Mór and Várpalota, G. T., 32, 20 (1986).

2. P. Varga, G. Szeidovitz, R. Gutdeutsch, Isoseismical map and tectonical position of the Komárom earthquake of 1763, A. G. G. Hung., 36, 12 (2001).
3. U. Eisinger, R. Gutdeutsch, C. Hammerl, Historical earthquake research, Abh. Geol. B.-A., 1, 19 (1992).

4. H. Ryu, J.K. Kim, J.W. Baker, A probabilistic method for the magnitude estimation of a historical damaging earthquake using structural fragility functions. Bull. Seism. Soc. of Am., 99, 18 (2009).

5. E.C. Morais, L.G. Vigh, J. Krähling, Fragility estimation and comparison using IDA and simplified macro-modelling of in-plane shear in old masonry walls, Spring. Proc. in Math. \& Stat., 181, 14 (2016).

6. S.A. Freeman, Development and use of capacity spectrum method, Proceedings of 6th US National Conference on Earthquake Engineering, 8 (1998).

7. E.C. Morais, L.G. Vigh, J. Krähling, A methodology for the development of Historical building archetypes for Seismic performance assessment, P. Periodica, 13, 12 (2018).

8. E.C. Morais, L.G. Vigh, J. Krähling, Historical earthquakes and historical building archetypes for dynamic structural analysis using historical surveys, $3^{\text {rd }}$ PROHITECH, 12 (2017).

9. F. Mckenna, G. Fenves, M. Scott, B. Jeremic, Open system for earthquake engineering simulation (OpenSees)S, (2006).

10. FEMA HAZUS-MH MR-1 Technical Manual, Fed. Emergency Management Agency, 699 (2003).

11. E.C. Morais, L.G. Vigh, J. Krähling, Seismic damage analysis of a Hungarian historical peasant house archetype, $9^{\text {th }}$ ENOC, 6 (2017).

12. FEMA P695 Quantification of building seismic performance factors, Fed. Em. Man. Ag., 216 (2009).

13. AHEAD, The Eur. Arch. of Hist. Earthquake Data, DOI: http://doi.org/10.6092/INGV.IT-AHEAD.

14. D. Vamvatsikos, C.A. Cornell, Incremental dynamic analysis, Earthq. Eng Struct. Dyn., 31, 24 (2002).

15. P.B. Lourenço, Computational strategies for masonry structures, PhD dissert., Delft University, 220 (1996).

16. S.H. Rafsanjani, A. Bakhshi, M.A. Ghannad, M. Yekrangnia, F. Soumi, Predictive tri-linear benchmark curve for in-plane behaviour of adobe walls, Int. Journ. of Arch. Herit., 9, 29 (2015).

17. S. Lagomarsino, S. Cattari, Perpetuate guidelines for seismic performance-based assessment of cultural heritage masonry structures, Bulletin of Earthquake Engineering, 13, 35 (2015).

18. S. Akkar, J.J. Bommer, Empirical equations for the prediction of PGA, PGV, and spectral accelerations in Europe, the Mediterranean Region, and the Middle East, Seismological Research Letters, 81, 12 (2010).

19. E.C. Morais, L.G. Vigh, J. Krähling, Preliminary estimation of the probable magnitude of Komárom 1763 earthquake using fragility functions, $16^{\text {th }}$ WCEE, 12 (2017). 